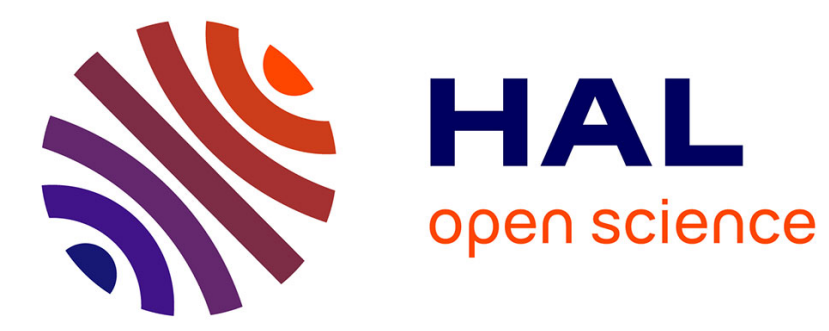

\title{
2D Visual Micro-Position Measurement based on Intertwined Twin-Scale Patterns
}

Valerian Guelpa, Patrick Sandoz, Cédric Clevy, Nadine Piat, Miguel Asmad Vergara, Guillaume Laurent

\section{- To cite this version:}

Valerian Guelpa, Patrick Sandoz, Cédric Clevy, Nadine Piat, Miguel Asmad Vergara, et al.. 2D Visual Micro-Position Measurement based on Intertwined Twin-Scale Patterns. Sensors and Actuators A: Physical , 2016, 248, pp.272 - 280. hal-03427080

\section{HAL Id: hal-03427080 https://hal.science/hal-03427080}

Submitted on 12 Nov 2021

HAL is a multi-disciplinary open access archive for the deposit and dissemination of scientific research documents, whether they are published or not. The documents may come from teaching and research institutions in France or abroad, or from public or private research centers.
L'archive ouverte pluridisciplinaire HAL, est destinée au dépôt et à la diffusion de documents scientifiques de niveau recherche, publiés ou non, émanant des établissements d'enseignement et de recherche français ou étrangers, des laboratoires publics ou privés. 


\title{
2D Visual Micro-Position Measurement based on Intertwined Twin-Scale Patterns
}

\author{
Valérian Guelpa ${ }^{\mathrm{a}}$, Patrick Sandoz ${ }^{\mathrm{b}}$, Miguel Asmad Vergara $^{\mathrm{b}, \mathrm{c}}$, Cédric Clévy $^{\mathrm{a}}$, Nadine Le Fort-Piat ${ }^{\mathrm{a}}$, \\ Guillaume J. Laurent ${ }^{\mathrm{a}}$ \\ ${ }^{a}$ Automation and Micro-Mechatronics Systems Department, FEMTO-ST Institute, Univ. Bourgogne Franche-Comté, UMR CNRS 6174, \\ ENSMM, UFC, Besançon, France \\ ${ }^{b}$ Applied Mechanics Department, FEMTO-ST Institute, Univ. Bourgogne Franche-Comté, UMR CNRS 6174, ENSMM, UFC, Besançon, \\ France \\ ${ }^{c}$ Sección Física, Departamento de Ciencias, Pontificia Universidad Católica del Perú, Apartado 1761, Lima, Peru.
}

\begin{abstract}
Position measurement at nanoscale currently raises issues such as making significant compromise between range and resolution or as the difficulty to measure several directions with a single sensor. This paper presents a novel visual method to measure displacements at nanometric scale along two axes. This method allows subpixelic measurement of position by using a pseudo-periodic pattern observed by a regular visual setup. This micrometric pattern corresponds to the intertwining of two perpendicular copies of a single-axis pattern made of two frequency carriers with slightly different periods. It was realized in clean room by photolythography of aluminium on glass. The algorithm is based on a twin-scale principle, itself based on direct phase measurement of periodic grids. Experiments are performed at video rate (30 fps) and show a linearity below $0.16 \%$ and a repeatability below $14 \mathrm{~nm}$ over an unambiguous range of $221 \mu \mathrm{m}$. A resolution below $0.5 \mathrm{~nm}$ is demonstrated by the use of 2000 images. The method can be adjusted to different ranges, according to the needs.
\end{abstract}

Keywords: displacement sensing; subpixelic; direct phase computation; two-axes pattern; nanometric precision.

\section{Introduction}

Position measurement raises specific issues in the microworld. Often the research of accuracy, range or overall dimensions needs compromises at this scale. Numerous sensors have the drawback to be in touch with the moving object (lot of capacitive or piezoelectric sensors for example) and are limited to one-direction measurement. Many sensors without contact, like interferometers or confocal sensors, are also limited to one direction. In this context, a measurement method by vision could be the solution of choice for 2D measurements.

A wide variety of visual methods exist and are able to detect sub-pixelic motions (typically with a resolution between 0.1 and 0.01 pixel). Some can be qualified as areabased methods: they work on the entire image without extracting particular features. It is also possible to use image features, for example with gradient-based motion estimation [1] or phase-correlation method like Huang et al. 2] who apply correlation to a coded but non-periodic structure and measure displacements with a resolution of $60 \mathrm{~nm}$ on a several millimeters range. Other methods use specific features in the image and measure their displacements, like Kim et al. who evaluate displacement of MEMS with the tracking of markers [3], or Kokorian et al. who detect picometric displacements with a curve-fitting based method [4.

Nevertheless these methods are limited in range by the size of the field of observation, that is why they only allow incremental measurements and hence a relative knowledge of the position. But another category of visual methods solves this problem: pattern-based methods have a range dependent on the used pattern, not on the field of observation.

With simple periodic patterns, it is possible to measure position accurately by feature-detection, as Clarck et al. [5], or by Fourier-like processing, as Yamahata et al. [6], but in these cases the absolute range is limited to one period. The most common solution to improve the range is to combine a large encoding to the periodicity. Various codes are usable, as the Manchester code used by Masa et al. [7] or a pseudo-periodic binary pattern for $2 \mathrm{D}$ measurement of Galeano-Zea et al. 8]. It is also possible to use moiré fringes to improve the range, as Ri et al. 9] or Sugiura et al. [10].

Another solution, inherited from interferometry, consists in using two fringe sets with different periods [11, [12. The combination of those two fringe sets forms a larger pattern while being an accurate tool. This method was already applied to displacement measurement along one direction in a previous work [13]. We also applied it to the measurement of a force through the use of a compliant structure [14. Experiments showed a $5 \mathrm{~nm}$ error for a range of $168 \mu \mathrm{m}$ and real-time processing.

This paper presents a novel visual method to measure displacements at nano-scale along two directions through 


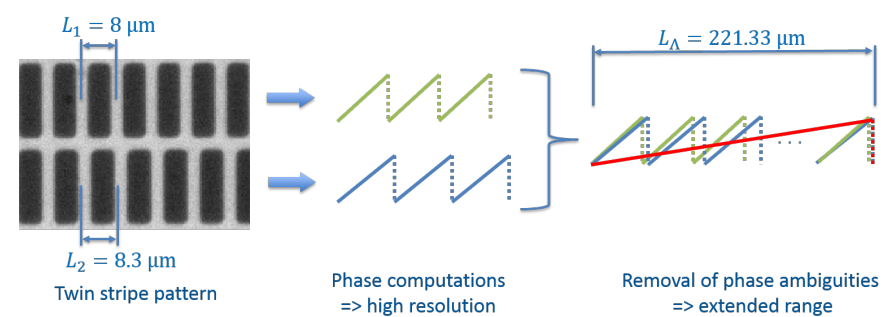

Figure 1: Working principle of the twin-scale visual measurement [14.

the observation of a single pattern. This pattern results from the intertwining of periodic grids that requires a specific algorithm to be used. The method combines accuracy and range along two-axes with monocular vision using a regular microscope.

The next sections present the principle of the method, firstly for 1-D measurement and then along two axes in section 3. Section 4 describes the experimental validation of the method and the obtained performances. Section 5 concludes the paper.

\section{Twin-scale measurement principle along one di- rection}

We previously presented a displacement measurement method based on pattern [13, [14. Despite a high performance level, this method is limited to one-direction measurement. The aim of this paper is to move beyond by doing 2D measurements with a similar principle. This section introduces the 1D measurement principle prior to develop the $2 \mathrm{D}$ method.

\subsection{Single-grid position measurement}

The use of a periodic pattern to evaluate the position of an object is not a new concept. A method consists in using phase measurement to obtain an estimation of the position, modulo the period of the pattern.

Fundamentally, the method is based on the phase-todisplacement relationship:

$$
\mathcal{F}(f(x-\delta))=e^{-2 \pi i \delta \xi} \cdot \mathcal{F}(f(x))
$$

where $\mathcal{F}$ corresponds to the Fourier transformation, $f(x)$ is the distribution of pixel intensity (or more generally the space function), $x$ the initial spatial coordinate, $\delta$ the spatial displacement and $\xi$ the reciprocal variable of $\mathrm{x}$. In this way, the spatial displacement $\delta$ induces a phase shift $\Phi$ in the frequency domain equal to $2 \pi \delta \xi$.

This relationship applied to an image $I$ of a periodic pattern allows to measure its displacement with high precision. Moreover, a short computation time could be obtained through the use of a single-frequency spectral computation applied to the spatial frequency of the pattern used, instead of a complete discrete Fourier transform. In

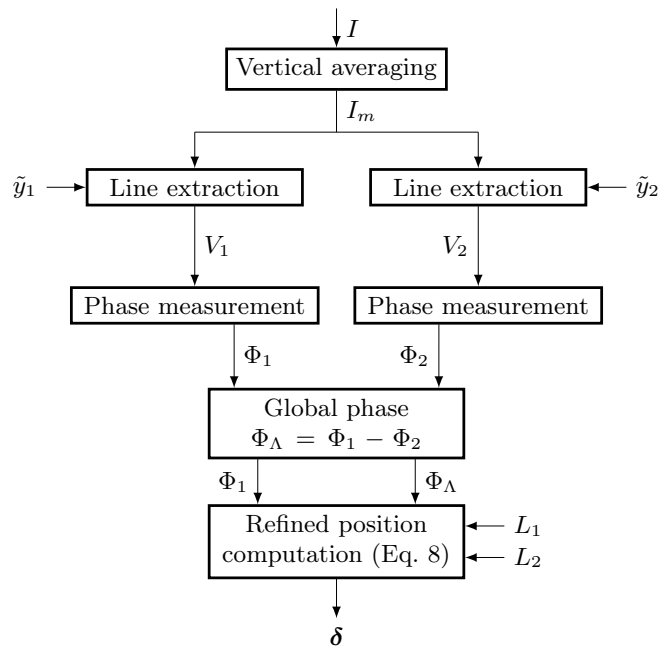

Figure 2: Block diagram of the twin-scale measurement method. Details are in 13 .

our case, it corresponds to calculate the phase $\Phi_{1}$ as the argument of the dot product between the pixel intensity vector $V$ (obtained after averaging of the image along the stripe direction) and a complex analysis vector $Z$ notably composed of a Gaussian window:

$$
\Phi_{1}=\arg (V \cdot Z)
$$

with :

$$
Z(k)=e^{-\left(\frac{k-\tilde{N} / 2}{\tilde{N} / 4.5}\right)^{2}} \cdot e^{-\left(\frac{2 i \pi(k-\tilde{N} / 2)}{\tilde{L}}\right)}
$$

with $k$ the pixel index, $\tilde{N}$ the image width in pixels and $\tilde{L}$ the stripe period in pixel: 1 obtained with a Fourier transform method.

The target displacement is determined with:

$$
\delta=\frac{\Phi_{1} \cdot L}{2 \pi}+n L
$$

where $n$ is an unknown integer and $L$ the period in meters. Indeed the pattern periodicity involves a modulo $L$ on the knowledge of its position by vision. Since a displacement equal to an entire number of periods does not change the image obtained.

\subsection{Twin-scale principle}

In order to improve the range of the method, we use a twin-scale pattern [13]. A second grid, with a slightly different period, is joined to the first grid. The phase measurement $\Phi_{2}$ of this grid provides another phase data that allows to remove phase ambiguities on the global pattern (see Fig. 1):

$$
\Phi_{\Lambda}=\Phi_{1}-\Phi_{2}
$$

\footnotetext{
1 In this article, spacial dimensions are metric by default. The dimensions in pixels are wrote with a tilde, like $\tilde{L}$.
} 
Table 1: Performances of twin-scale visual measurement with a $8 \mu \mathrm{m}$ periodic pattern and 640x480 8-bits camera (from [13]).

\begin{tabular}{lc}
\hline Property & Value \\
\hline Travel range & $168 \mu \mathrm{m}$ \\
Theoretical resolution & $55 \mathrm{pm}$ \\
Experimental repeatability $(3-\sigma)$ & $5 \mathrm{~nm}$ \\
Bandwidth & $>1500 \mathrm{~Hz}$ \\
\hline
\end{tabular}

The synthetic period of the pattern is given by:

$$
L_{\Lambda}=\frac{L_{1} \cdot L_{2}}{\left|L_{1}-L_{2}\right|}
$$

where $L_{1}$ and $L_{2}$ are the periods of the two strip sets. The accuracy of the method is also improved by a calculus trick: the position is not directly evaluated with the phase $\Phi_{\Lambda}$ but with $\Phi_{1}$ and the entire number $m$ of periods $L_{1}$ in the period $L_{\Lambda}$ :

$$
m \approx \frac{L_{\Lambda} \Phi_{\Lambda}-L_{1} \Phi_{1}}{2 \pi L_{1}}
$$

Finally the displacement is given by:

$$
\delta=\frac{\Phi_{\Lambda} L_{\Lambda}}{2 \pi}+p L_{\Lambda}=\frac{L_{1}}{2 \pi} \Phi_{1}+m L_{1}+p L_{\Lambda}
$$

where $p$ is an unknown integer (can be set to 0 if the range of displacement is below $\left.L_{\Lambda}\right)$. The new ambiguity is equal to $L_{\Lambda}$ rather than $L_{1}$. The algorithm is summarized Fig. 2,

When the periods $L_{1}$ and $L_{2}$ of the patterns are known, the method provides a direct conversion from pixels to meters. This self-calibration does not depend on experimental parameters such as magnification or field of view. High-frequency noises are also filtered by the spectral filtering involved in the phase measurement. The method presents low sensitivity to sharpness or brightness variation, at least as long as the contrast is sufficient to extract spacial frequencies of interest. The determinant parameter to obtain accuracy is finally the resolution of the camera (see [13]).

Table 1 gathers the performances of the method using a micro-machined pattern with twin-scales of periods $8 \mu \mathrm{m}$ and $8.4 \mu \mathrm{m}$. The range-to-resolution ratio of the method is larger than $10^{6}$ with a 8 -bits camera. Its working principle and the evaluation of these performances are detailed in 13 as well as discussions about the influence of the parameters.

\section{Extension to two-direction measurement}

In view of performances obtained for one axis displacement measurement, we worked on the evolution of the method to be able to measure displacements along two axes with the objective of similar performances in terms of range and accuracy.

Basically, a 2D measurement could be done through the use of two 1D sensors correctly placed. In the case of the method previously exposed, two patterns could be placed perpendicularly on the object of interest and observed by two cameras during a planar displacement, what would result in $2 \mathrm{D}$ measurement. However in numerous cases, and more particularly in the micro-world, the bulk of the sensing setup is a critical point. That is why a single $2 \mathrm{D}$ sensor is preferable to a pair of $1 \mathrm{D}$ sensors.

The use of the new pattern presented below allows to carry out direct $2 \mathrm{D}$ measurement with a single camera. This section introduces this pattern and describes the measurement process. This process is divided in two points: the measurement algorithm itself and the necessary preprocessing steps.

\subsection{Pattern design}

A new pattern was designed and micro-machined in clean room to measure displacements along two directions simultaneously. Its shape results from the fusion of two twin-scale patterns intertwined perpendicularly by inclusive disjunction (see Fig. 3). The result is a chessboardlike pattern that stores the entire data of the two previous patterns and suits for measurement displacements with a large unambiguous range along the two axes.

In the experimental case $L_{x, 1}=L_{y, 1}=L_{1}=8 \mu \mathrm{m}$ and $L_{x, 2}=L_{y, 2}=L_{2}=8.3 \mu \mathrm{m}$, resulting in an unambiguous range of $221.33 \mu \mathrm{m}$. The heights $W_{x}$ and $W_{y}$ of the pattern are larger than the periods $L(32 \mu \mathrm{m})$ with the aim to not affect measurements. It is noteworthy that these periods can be adapted to the needs.

In practice, the pattern is realized by photolithography on a thin aluminum layer deposited on a glass substrate (see Fig. 3 c). The elementary, non-ambiguous range of $221.33 \times 221.33 \mu^{2}$ is duplicated several times in both directions and the actual full pattern size is $10 \times 10 \mathrm{~mm}^{2}$.

\subsection{Algorithm}

The new pattern requires a new algorithm to be used. Both horizontal and vertical displacements have to be measured by phase evaluation applied to specific areas of the image, similarly to the method presented in section 2.1. The major problem is now the choice of these areas. Only the case of horizontal measurement will be detailed here since the vertical one is similar. The global measurement algorithm, detailed below, is presented in Fig. 5 .

The first step consists in the vertical filtering of the image:

$$
I_{m}=I * C
$$

with $C$ a column vector of size $\tilde{f}_{y}=\operatorname{round}\left(\tilde{L}_{y, 1}+\tilde{L}_{y, 2}\right) / 2$ with each component equal to $1 / \tilde{f}_{y}$. The aim of this step is to cancel high frequency noises and recover a 1D-pattern shape in the 2D-pattern image (see Fig. 4). 


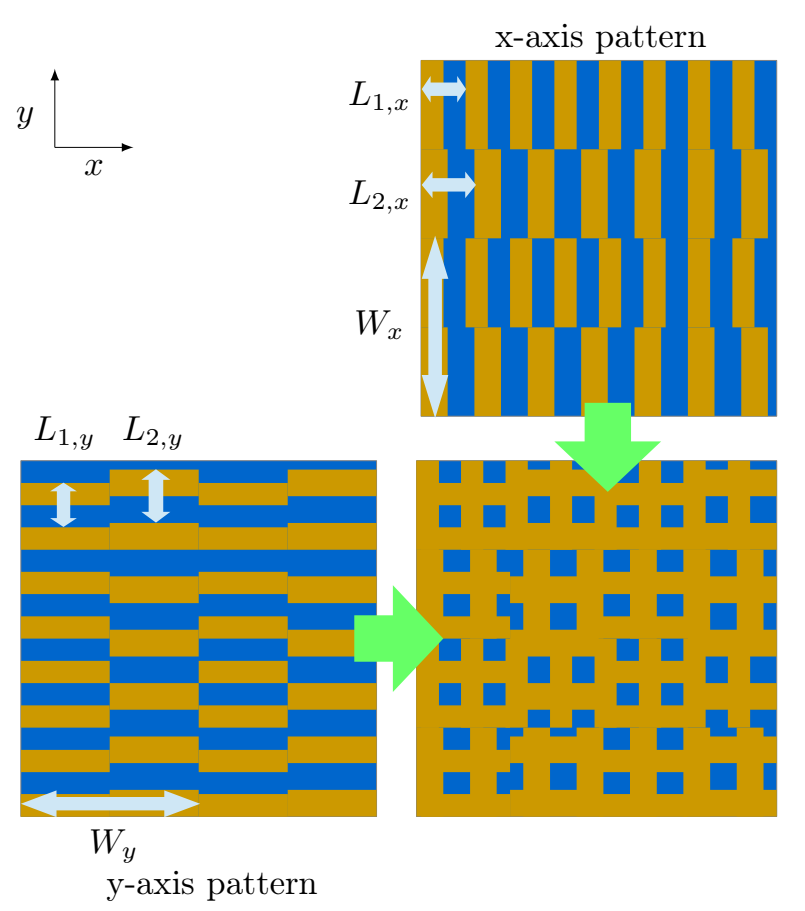

(a) Design principle of the 2D pattern.

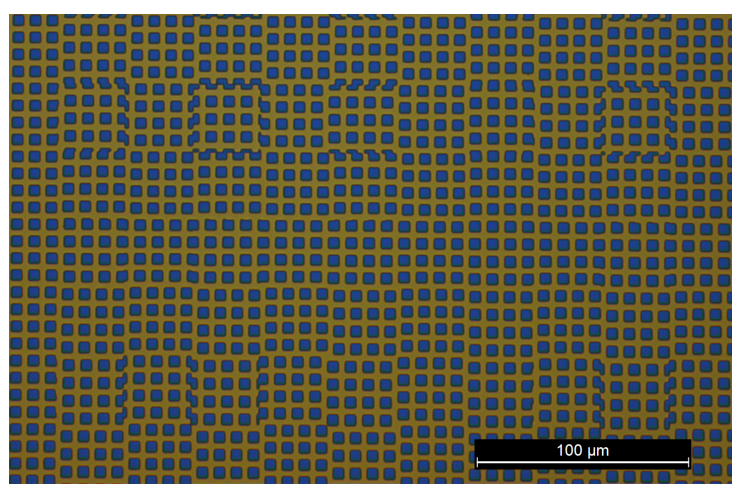

(b) Mask used for the photolithography of the pattern in clean room. Here $L_{x, 1}=L_{y, 1}=8 \mu \mathrm{m}, L_{x, 2}=L_{y, 2}=8.3 \mu \mathrm{m}$ and $W_{x}=W_{y}=32 \mu \mathrm{m}$.

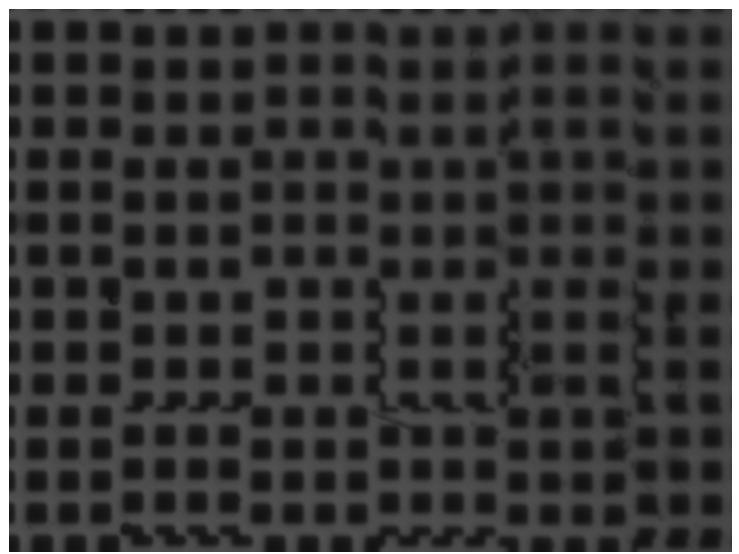

(c) Typical 1280x960 image of the pattern captured with the visual setup.

Figure 3: The 2D pattern, from principle to actual device.

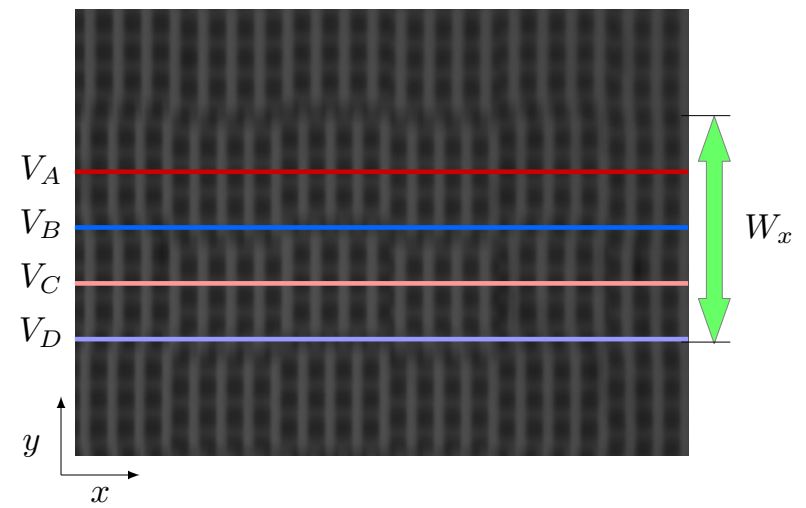

Figure 4: An example of selection of four horizontal lines used to find the best working area. The image is averaged vertically beforehand. Each line is chosen $\tilde{W}_{x} / 4$ away from the previous line.

The second step consists in the extraction of four horizontal and equidistant lines, named $V_{A}, V_{B}, V_{C}$ et $V_{D}$ (see Fig. (4). The aim of these multiple lines is to identify a couple of lines corresponding to periods $L_{1}$ and $L_{2}$ respectively. Indeed at least one of the couples $A C$ or $B D$ is suitable, i.e. without stripe set overlapping, because of the choice of a $W / 4$ distance between these lines.

Then eight products are then computed:

$$
P_{K i}=\sum_{\tilde{x}} V_{K}(\tilde{x}) \cdot Z_{x, i}\left(\tilde{x}, \tilde{L}_{x, i}\right)
$$

with $K \in[A, B, C, D], i \in[1,2]$ and $Z_{x, i}$ the analysis function (similar to that of equation 3 ) such as:

$$
Z_{x, i}\left(\tilde{x}, \tilde{L}_{x, i}\right)=e^{-\left(\frac{\tilde{x}-\tilde{N} / 2}{\tilde{N} / 4.5}\right)^{2}} \cdot e^{-\left(\frac{2 i \pi(\tilde{x}-\tilde{N} / 2)}{\tilde{L}_{x, i}}\right)}
$$

To determine which is the best couple and who is who in this couple (who corresponds to period $L_{x, 1}$ and who corresponds to period $L_{x, 2}$ ), four cross-modulus are calculated:

$$
\begin{aligned}
M_{A 1 C 2} & =\left|P_{A 1}\right| \cdot\left|P_{C 2}\right| & M_{A 2 C 1} & =\left|P_{A 2}\right| \cdot\left|P_{C 1}\right| \\
M_{B 1 D 2} & =\left|P_{B 1}\right| \cdot\left|P_{D 2}\right| & M_{B 2 D 1} & =\left|P_{B 2}\right| \cdot\left|P_{D 1}\right|
\end{aligned}
$$

The largest cross-modulus corresponds to the best couple, noted $M_{\text {best }}$ (since stripe overlapping or period mismatch would reduced the modulus drastically).

The penultimate step consists in calculating the phases of the two grids of the chosen couple and to correct them to take into account the slight residual misalignment between the pattern and the camera pixel frame (see Fig. 5). It requires the rate $\theta_{\text {rad } / p, x}$ of the rotation of the pattern with respect to the pixel frame (in radian per pixel). For example if $M_{\text {best }}=M_{B 1 D 2}$, we know that $V_{B}$ has a period $L_{1}$ and $V_{D}$ has a period $L_{2}$, so we obtain:

$$
\begin{aligned}
& \phi_{B 1}=\arg \left(P_{B 1}\right)+\left(\tilde{y}_{A}-\tilde{y}_{B}\right) \cdot \theta_{\text {rad } / p, x} \\
& \phi_{D 2}=\arg \left(P_{D 2}\right)+\left(\tilde{y}_{A}-\tilde{y}_{D}\right) \cdot \theta_{\text {rad } / p, x}
\end{aligned}
$$

Finally these two phases are used to determinate the position with the same method as for one degree of free- 
dom measurement (see equation 8). The same algorithm is applied to the y-axis.

It is important to mention that some parameters have to be identified before the measurement: the periods of the pattern (in pixel) $\tilde{L}_{x, 1}, \tilde{L}_{x, 2}, \tilde{L}_{y, 1}, \tilde{L}_{y, 2}$; its heights $\tilde{W}_{y}$ and $\tilde{W}_{x}$; the analysis functions $Z_{x, 1}, Z_{x, 2}, Z_{y, 1}$ and $Z_{y, 2}$; the rates $\theta_{r a d / p, x}$ and $\theta_{r a d / p, y}$. These parameters are determined once by a pre-processing step, as described with full details in next section.

\subsection{Parameters identification}

The pre-processing step allows to evaluate ten elements useful for the measurement step: the four grid periods, the two grid widths, the four analysis functions and the two angular corrections. The global calibration algorithm is shown in Fig. 6 for the half of the elements (the second half is obtained similarly by inverting $\mathrm{x}$-axis and $\mathrm{y}$-axis.

In a first instance, the pre-processing is made on filtered images (as the measurement step), allowing to reduce high frequency noises (see equation 9). The four periods in pixels $\left(\tilde{L}_{x, 1}, \tilde{L}_{x, 2}, \tilde{L}_{y, 1}, \tilde{L}_{y, 2}\right)$ are firstly determined thanks to a Fourier transform applied to the selected vectors. Next an iterative algorithm allows to find these periods with subpixelic resolution (inferior to 0.01 pixel). The analysis functions $Z_{x, 1}, Z_{x, 2}, Z_{y, 1}$ and $Z_{y, 2}$ are calculated thanks to these periods (see equation 11).

The widths of the grids $\left(\tilde{W}_{x}, \tilde{W}_{y}\right)$ are obtained with the same algorithm but applied to vectors made by specific filtering of the image to bring to light the widths.

Finally the necessary angular correction is calculated by considering the deviation of the phase variation, horizontally or vertically. For example if the rows of an image are scanned to measure the horizontal position of the pattern according to the ordinate, the phase curve is a line tilted by the angle of the pattern. Thus the phase error $\theta_{\text {rad } / p, x}$ (in radian by pixels) due to the orientation of the pattern can be calculated. However this evaluation of the orientation is limited to small angles, due to residual static misalignment.

Globally most of the pre-processing time corresponds to the selection of four rectangular areas by the operator to obtain the coordinates $\tilde{x}_{1}, \tilde{x}_{2}, \tilde{y}_{1}$ and $\tilde{y}_{2}$. The size of the filter applied to the image $\left(\tilde{f}_{x}\right.$ and $\left.\tilde{f}_{y}\right)$ can also be set. This time is of course user-dependent and it was evaluated to be close to $30 \mathrm{~s}$ by averaging from different users (extremes $20 \mathrm{~s}-45 \mathrm{~s}$ ). In comparison, the actual preprocessing computation time of $1.3 \mathrm{~s}$ is almost negligible.

\section{Experimental validations}

An experimental setup was built to test the method (see Fig. 7). It primarily consists of a pattern fixed on a nano-stage and observed through a microscope. Evaluated performances are resolution, range, linearity, repeatability and trueness.

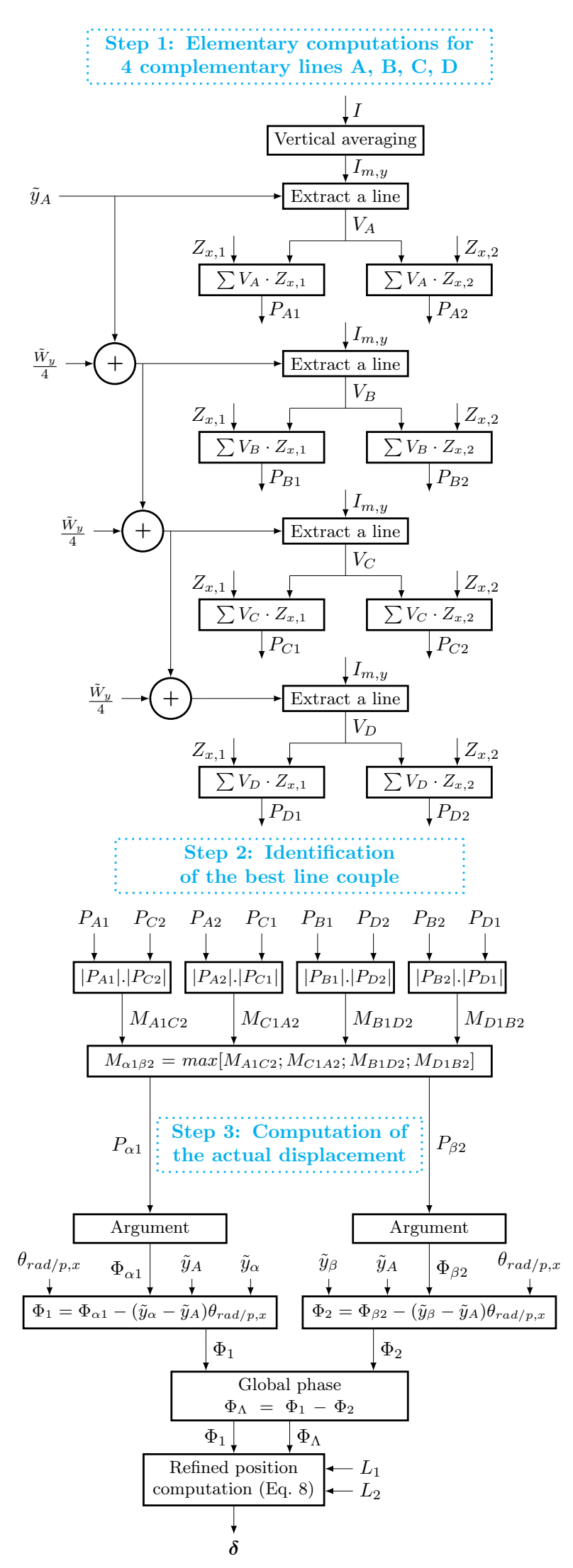

Figure 5: Block diagram of 2D measurement. Here only the measurement along the $\mathrm{x}$-axis is presented, but the $\mathrm{y}$-axis case is similar. 


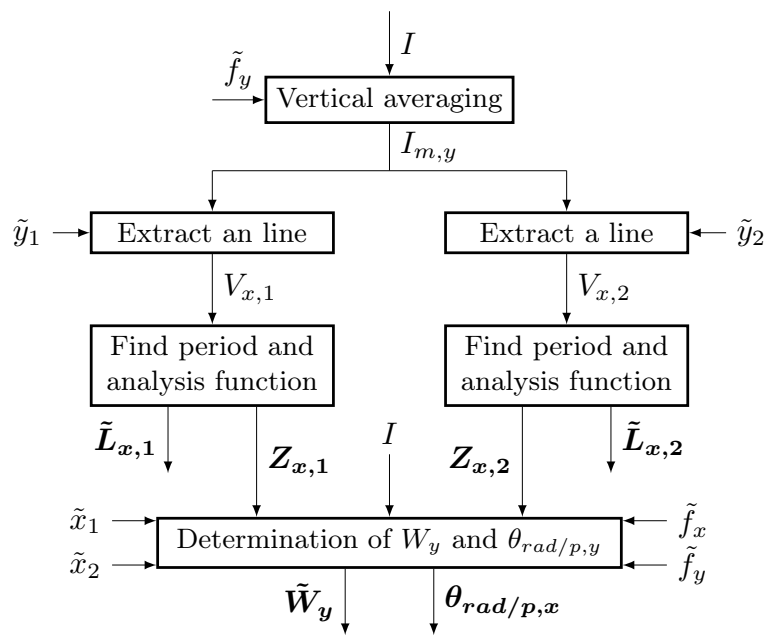

Figure 6: Pre-processing block diagram to identify accurately parameters. A symmetrical algorithm allows to find the complementary parameters.

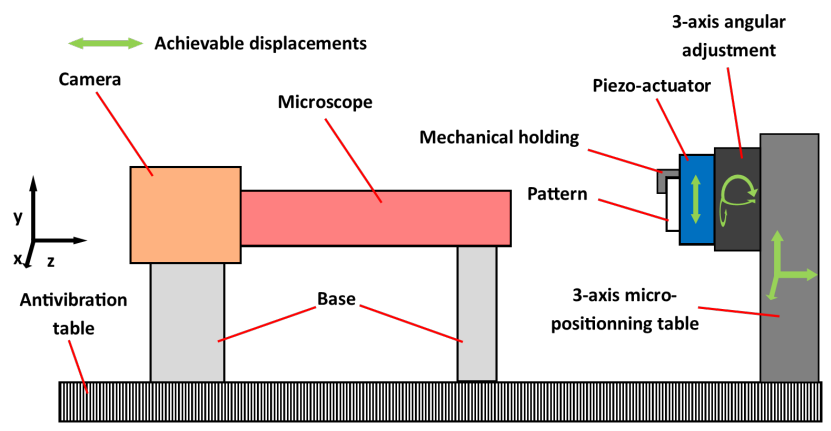

(a) Diagram of the experimental setup. The pattern could be moved along six DoF manually and one DoF automatically.

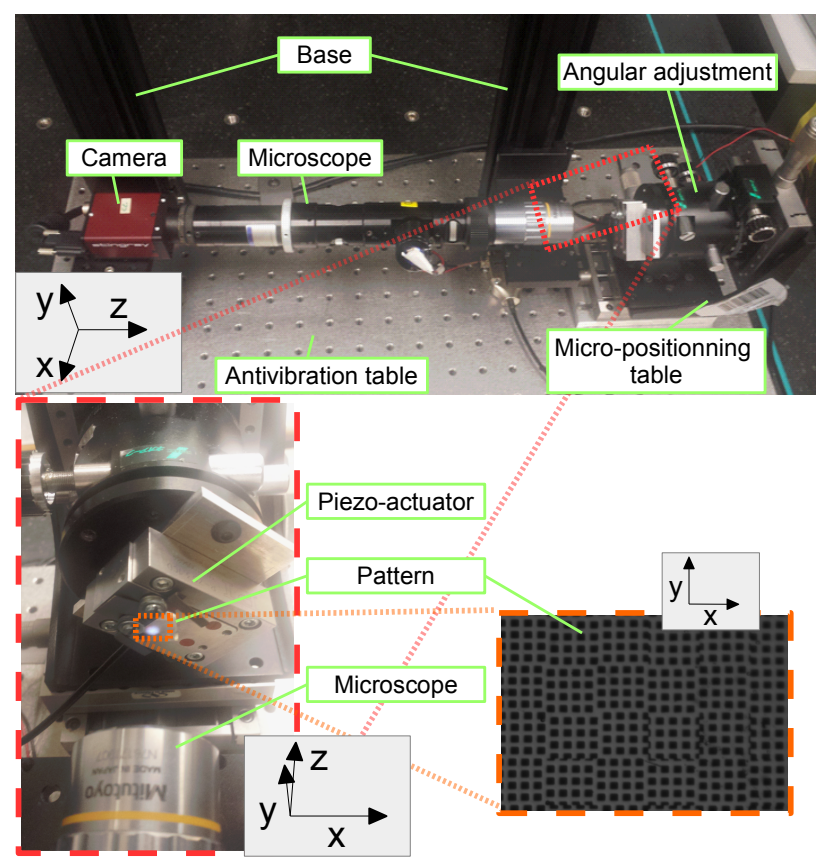

(b) The experimental setup from various points of view.

Figure 7: The experimental setup, placed in a metrological room to reduce mechanical and thermal noises. The 1 DoF piezo-actuator is placed obliquely to generate a pattern displacement along both $\mathrm{x}$ and y axes.

\subsection{Experimental setup}

The aim of the experimental setup is to observe a moving pattern to evaluate the performances of the method, thus it is important to control precisely the displacement. We use for this purpose a piezo nanopositioner P-753.1CD from Physik Instrumente (specifications in table 3).

This piezoactuator is mounted on a 6 -axis manual positioning device (three translations and three rotations) allowing micro-displacements. This device is used to place the pattern in front of the microscope without out-of-plane tilt and to realize displacements larger than the piezoactuator range.

The visual setup is composed of a Fireware camera (Allied Vision Technology Stingray F-125, 8 bits, $1292 \times$ 964 pixels) and a $10 \times$ microscope lens. The computer used is a common Intel Core2 Quad CPU Q9550 2.83 GHz, running under Windows 7 .

The performances of the visual measurement method are determined by observing the pattern fixed on the actuator, so these performances are highly dependent on noises that move the pattern with respect to the microscope. To reduce mechanical noises, the setup is fixed on an antivibration table placed in a metrologic room with an isolated slap. Thermal noises are reduced by the temperature control of the room (variations below 0.5 degrees).

\subsection{Resolution evaluation}

In this work, we refer to resolution definition provided by the "Joint Committee for Guides on Metrology" 15] as the "smallest change in a quantity being measured that causes a perceptible change in the corresponding indication". So the important is to detect a displacement as small as possible, even if it is lost within a noisy background of much larger magnitude. In this way, the experiment sends a picometric control input to the piezoactuator and detects it with the vision (at 20 frames per second) to evaluate the resolution.

The chosen input signal is a pulse sequence of period $1 \mathrm{~s}$ with an amplitude of $0.5 \mathrm{~nm}$. This amplitude is near to the minimal displacement we can observe with our visual method, as demonstrated in [13. However a so little displacement will be lost in the measurement noise (here approximately $5 \mathrm{~nm}$, as observed in the following experiments). To detect the displacement despite noises, we use the Fourier transform applied to the temporal data of position measurement, to detect the periodicity of this sequence of displacement. Indeed if the peak of the resulting curve is found at the expected frequency, the displacement is detected so the visual method has a resolution at least equal to its amplitude.

Fig. 8. a shows the control input (with an amplitude of $0.5 \mathrm{~nm}$ ) and the corresponding capacitive sensor measurement. We can clearly see the repeatability of the capacitive is around $1 \mathrm{~nm}$. We can also check the effective motion by averaging the measures of low and high levels. We found a difference of $0.4937 \mathrm{~nm}$. Since the resolution of the capacitive sensor is ten times lower, we are confident that 


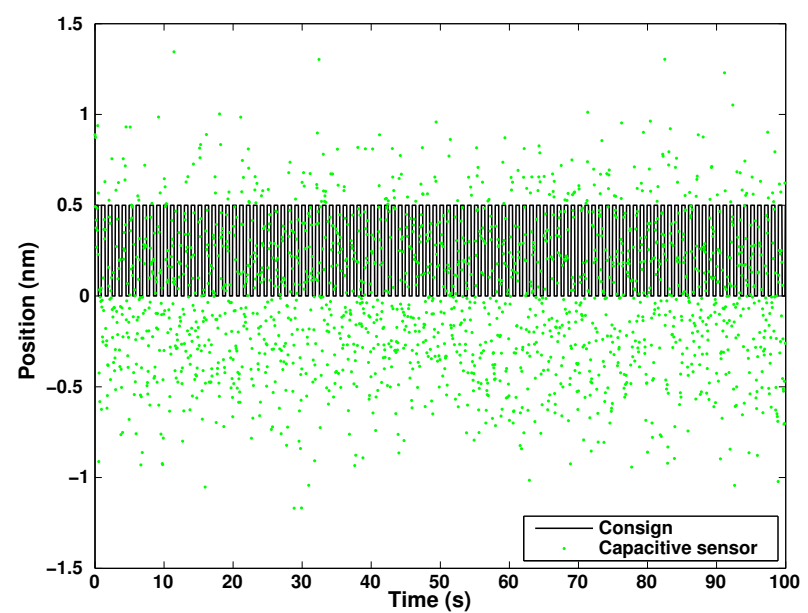

(a) The input command is a pulse of period $1 \mathrm{~s}$.

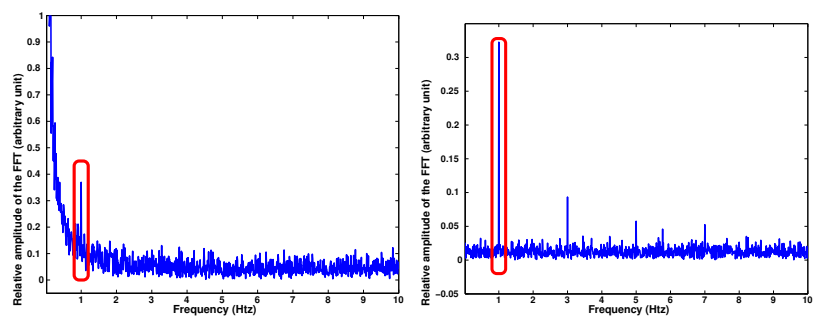

(b-c) Magnitude of the FFT of visual measurement along x-axis (left) and from the capacitive sensor measurement (right). The peak at frequency of $1 \mathrm{~Hz}$ is perceived more easily with the capacitive sensor because the signal is less noisy.

Figure 8: Results of the resolution experiment. The fact that the magnitude of FFT of both visual method and capacitive sensor have a peak which corresponds to the period of the input command proves that the resolution of the method is at least $500 \mathrm{pm}$.

a motion of $0.5 \mathrm{~nm}$ is produced. The question is then to detect the motion with the visual method. Fig. 8 b and c illustrate respectively the magnitude of the FFT of the temporal visual measurement along the $\mathrm{x}$-axis and of the capacitive sensor. We can see distinctly the peaks for a frequency of $1 \mathrm{~Hz}$, so the resolution of the visual method is (at least) equal to $0.5 \mathrm{~nm}$. This value is to be considered only with this pattern and this visual setup, for an analysis using 2000 images (theoretical best values for other setups are discussed in [13]). This result is widely below the others performances evaluated experimentally further (linearity, repeatability, trueness), and should thus be seen as an absolute measurement limitation in our experimental conditions.

\subsection{Micrometric measurements}

The second experiment is thought to allow the evaluation of the range, linearity, repeatability and trueness of the method. The input command is a ramp function (see Fig. 9). The pattern is always placed tilted beside the actuator to allows measurements simultaneously along $\mathrm{x}$-axis and $\mathrm{y}$-axis (see Fig. 7).

One series of measurements is presented in details below, but five others were carried out with the same proce-

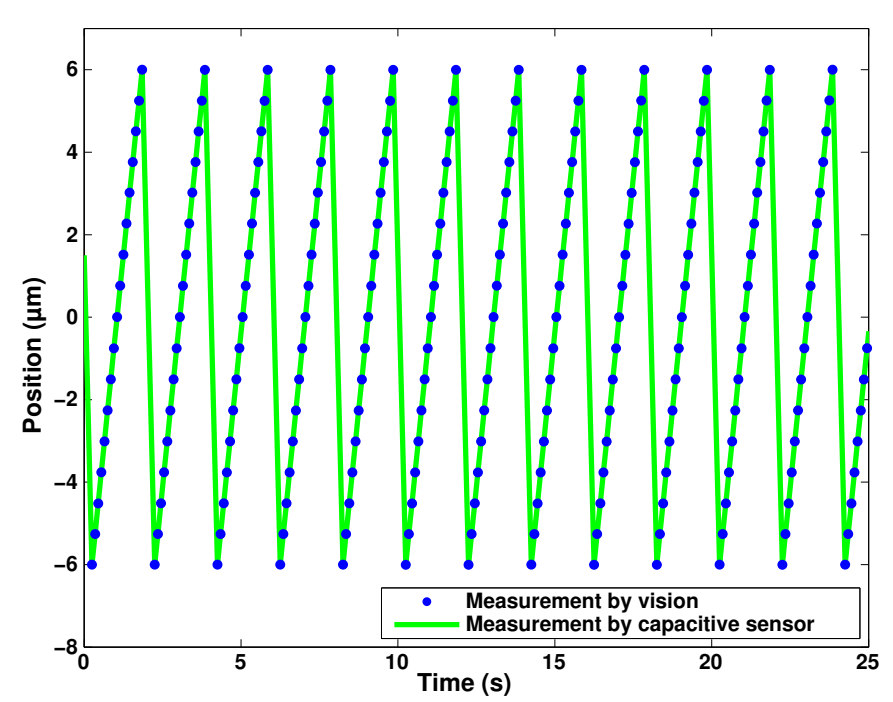

Figure 9: Typical position measurement during the micrometric experiment (here for serie 1, see Fig. 14). The input command is a ramp function of amplitude $12 \mu \mathrm{m}$. The global series is ten times longer than what is illustrated by the figure, with 2000 points.

dure. Each series includes 2000 points allocated between the 17 positions of a ramp function. The results are presented at the end of this section: Table 2 gives a general vision of these results; Table 3 summarizes these results and collates them to the performances of the piezo-actuator and its capacitive sensor. Also notable is that the calculation method is able to process more than 100 image per second under Matlab, meaning video-rate processing with common cameras.

\subsubsection{Accuracy estimation}

We choose to characterize the accuracy of our method with three elements: its linearity, its repeatability and its trueness.

The linearity is defined as the maximum deviation of the measured quantity values from the least square line of the measured values during a linear displacement. Fig. 10 shows the corresponding error values and the resulting linearity of $19 \mathrm{~nm}$, which corresponds to $0.16 \%$ relatively to the explored range during this experiment.

The repeatability (or measurement precision under repeatability conditions) is defined as the "closeness of agreement between indications or measured quantity values obtained by replicate measurements on the same or similar objects under specified conditions" 15]. Fig. 11 expresses this definition by the evaluation of three times the standard deviation of the measurement for each input command. The result is a repeatability below $10.5 \mathrm{~nm}$.

The trueness is defined as the "closeness of agreement between the average of an infinite number of replicate measured quantity values and a reference quantity value" [15]. In our case the reference is the capacitive sensor included 


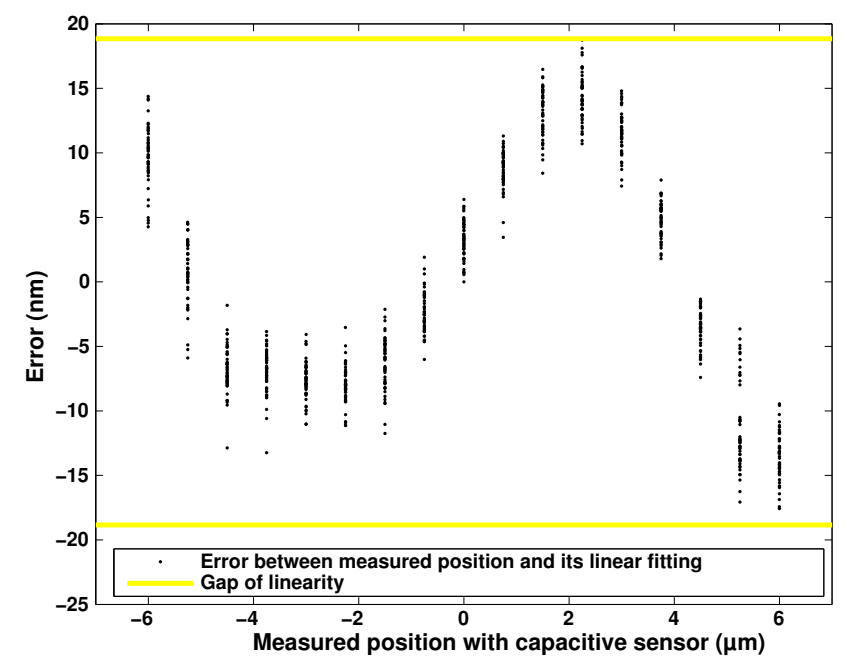

Figure 10: Error between the visual measurement and its least square line (serie 1, see Fig. 14). The linearity is the maximum deviation of this error, here equal to $19 \mathrm{~nm}$.

in the piezo-actuator and each trueness value is the average of more than hundred errors. Fig. 12 presents these trueness according to the input command, never bigger than $18.5 \mathrm{~nm}$. The accuracy of the actuator is better than that of visual sensor; in this way the wavy shape is due to edge effect on the pattern that induce a numerical error on the phase measurement.

\subsubsection{Range evaluation}

The range of the method is determined by the periods of the pattern according to Eq. 6. Thus with $L_{1}=8 \mu \mathrm{m}$ and $L_{2}=8.3 \mu \mathrm{m}$ the complete range is equal to $L_{\Lambda}=$ $221.33 \mu \mathrm{m}$. To illustrate this range, two 1D-axis with a larger range (M-111.1DG from Physik Instrumente) are used to move the pattern along the two axes. Their control inputs are sine curves with slightly different periods, what generates the trajectory of Fig. 13 .

To illustrate the fact that performances are homogeneous along the whole range, the initial setup (with the more accurate actuator) is used. The manual positioning table was used to change the $\mathrm{x}, \mathrm{y}$ and $\theta$ positions, then five measurement series (similar to that one used to evaluate accuracy) are done. Fig. 8 shows the 6 trajectories of the pattern and the maximal range. Results are summarized in Table 2 and Table 3 .

To conclude on the range issue, it is important to mention that displacements larger than $L_{\Lambda}$ can be measured by performing phase compensation; i.e. adjusting parameter $p$ in equation 8 . In this case the global range of the method is limited by the size of the pattern. It is a common method used to improve the range of application of a sensor.

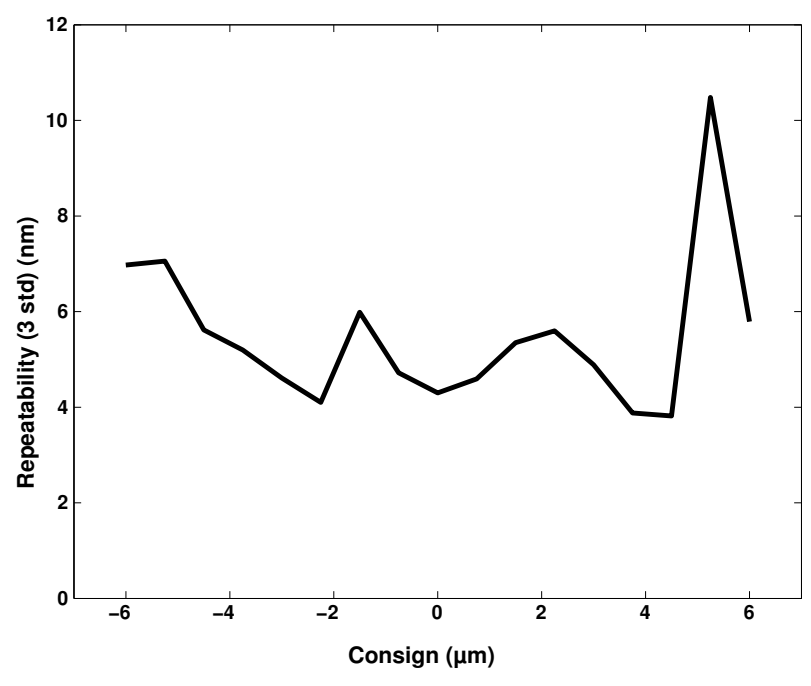

Figure 11: Repeatability of the visual measurement (serie 1, see Fig. 14. The repeatability is equal to $3 \sigma$ (with $\sigma$ the standard deviation), calculated with all the measures obtained for a specific input command. It corresponds coarsely to the dispersion of each group of point in Fig. 10 Here the repeatability is always below $10.5 \mathrm{~nm}$ and on average equal to $5.5 \mathrm{~nm}$.

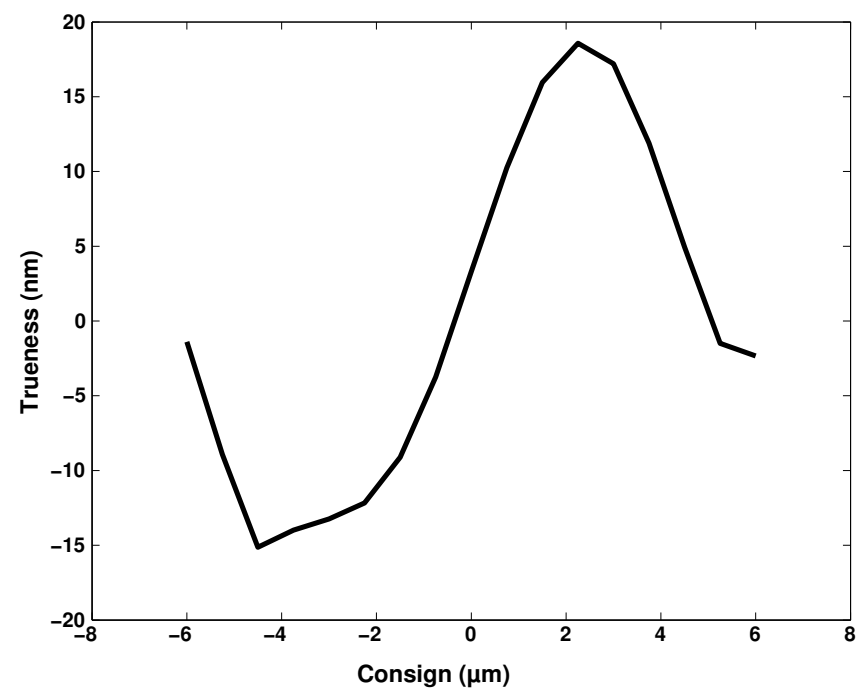

Figure 12: Trueness of the visual measurement (serie 1, see Fig. 14. Each trueness value is the error between the average of visual measurements and the average of capacitive measurements for a specified input command. Here the default of trueness is mainly due to the linearity default (similar to Fig. 10.

Table 2: Overview of the experimental results for the six series of measurements by vision.

\begin{tabular}{c|ccccccc}
\hline Performances & Serie 1 & Serie 2 & Serie 3 & Serie 4 & Serie 5 & Serie 6 \\
\hline Linearity (\%) & 0.16 & 0.13 & 0.16 & 0.12 & 0.12 & 0.16 \\
Repeatability (nm) & 10.5 & 10 & 13.5 & 6.5 & 12 & 11 \\
Trueness (nm) & 18.5 & 11 & 11 & 10 & 8 & 15 \\
\hline
\end{tabular}




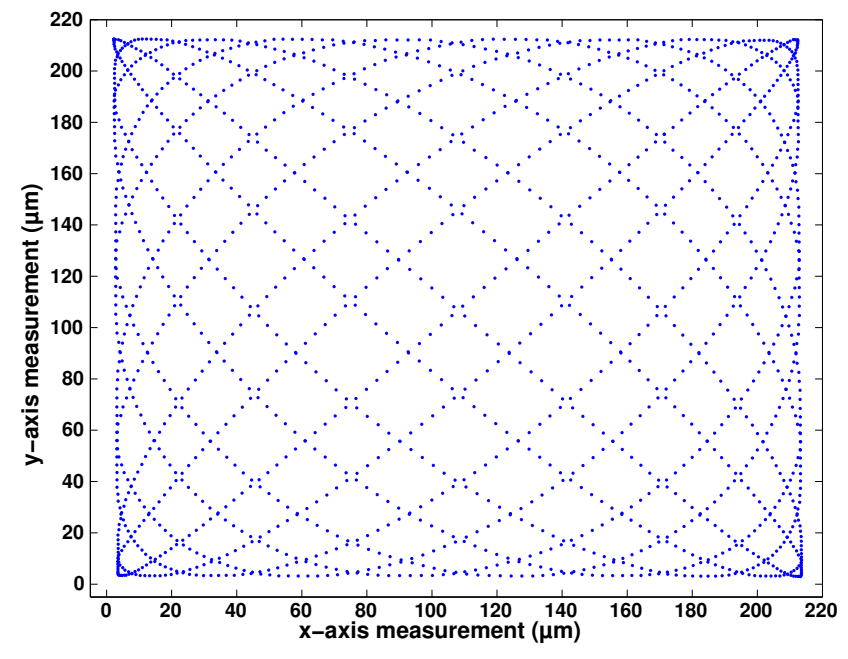

Figure 13: Illustration of the range of the visual sensor during a large-range measurement. Two actuators are used with sinusoidal control inputs.

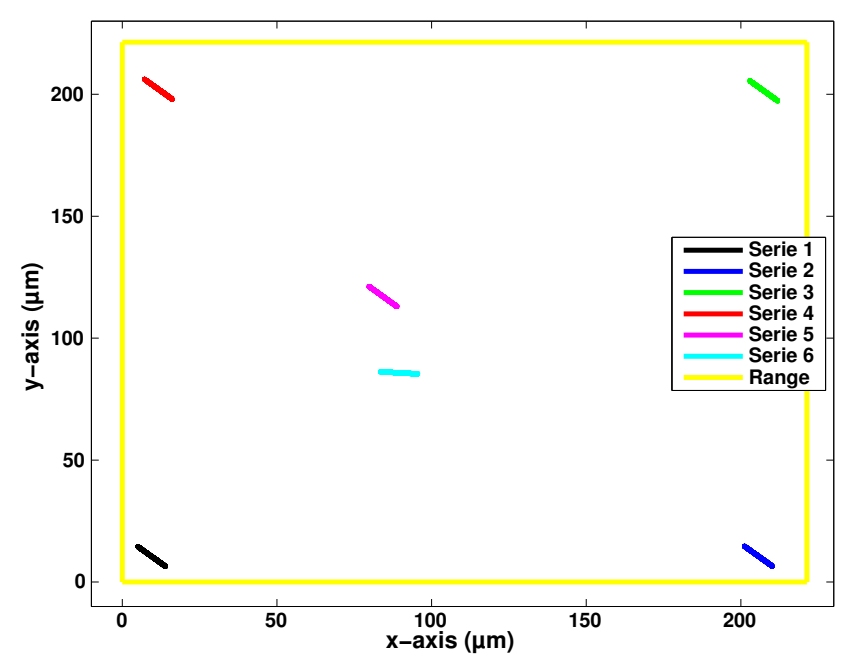

Figure 14: Trajectory of the six experimental series. Each serie is composed of 2000 measurements. Series 1 to 5 allow to test the range. Serie 6 supplied results when the displacement is mainly done along one axis (here $\mathrm{x}$-axis)

Table 3: Summary of results and comparison with the piezo-actuator performances.

\begin{tabular}{c|ccc}
\hline Performance & \multicolumn{2}{c}{ Visual method } & Capacitive sensor \\
& Worst case & Average value & \\
\hline Resolution (nm) & 0.5 & 0.14 & 0.05 \\
Linearity (\%) & 0.16 & 7.22 & 0.04 \\
Repeatability (nm) & 13.5 & 6.9 & 1 \\
Trueness (nm) & 18.5 & reference \\
Range ( $\mu \mathrm{m})$ & \multicolumn{2}{|c}{221.33} & 15 \\
\hline
\end{tabular}

\section{Conclusion}

The visual measurement method presented in this paper provides a satisfactory solution to the initial issue, namely the $2 \mathrm{D}$ position measurement with a large rangeto-resolution ratio. The new micro-pattern intertwines properly the twin-scale grids along the two axes. The new algorithm is adapted to its exploitation, selecting correctly which areas of the image are suited to realize measurements.

We experimentally characterized the performances of the method with a specific setup. Large absolute range $(221.33 \mu \mathrm{m})$, sub-nanometric resolution $(0.5 \mathrm{~nm})$ and good linearity $(0.16 \%)$, repeatability $(13.5 \mathrm{~nm})$ and trueness $(18.5 \mathrm{~nm})$ are observed (with a capacitive sensor as reference). Moreover the computation time enables video-rate use. The method constitutes thus a new tool for the calibration of micro-actuators as well as a supplementary visual sensor in the field of automation at micro-scale. The method is also easily and quickly calibrated. Finally it is important to mention that the visual method could be adapted to others scales: general enlargement or shortening of the pattern; modifications of $L_{x, 1}, L_{y, 1}, L_{x, 2}$ or $L_{y, 2}$ to modify its resolution or range; using of a more powerful camera and adjustment of the lens magnification accordingly; etc.

Future works will be focused on the use of a similar pattern to realize spatial position measurement, always aiming large range-to-resolution ratio.

\section{Acknowledgments}

This work was supported by Labex ACTION project (ANR-11-LABX-01-01) and by Région de Bourgogne-Franche-Comté. Authors acknowledge the French RENATECH network through its FEMTO-ST technological facilities MIMENTO, ROBOTEX and ENSMM for its metrological room. M. Asmad Vergara was supported by Student Grant Huiracocha Program-PUCP (Peru).

[1] Q. Lu, X. Zhang, and Y. Fan, "Micro-Vision-Based Displacement Measurement with High Accuracy," in Seventh International Symposium on Precision Engineering Measurements and Instrumentation, vol. 8321, 2011.

[2] W. Huang, C. Ma, and Y. Chen, "Displacement Measurement with Nanoscale Resolution using a Coded Micro-Mark and Digital Image Correlation," Optical Engineering, vol. 53, no. 12, pp. 124 103-1:7, 2014.

[3] Y.-S. Kim, S. Ho Yang, K. Woong Yang, and N. G. Dagalakis, "Design of MEMS Vision Tracking System Based on a Micro Fiducial Marker," Sensors and Actuators A: Physical, vol. 234, pp. 48-56, 2015.

[4] J. Kokorian, F. Buja, and W. M. van Spengen, "In-Plane Displacement Detection with Picometer Accuracy on a Conventional Microscope," Journal of Microelectromechanical Systems, vol. 24, no. 3, pp. 618-625, 2015.

[5] L. Clark, B. Shirinzadeh, U. Bhagat, and J. Smith, "A VisionBased Measurement Algorithm for Micro/Nano Manipulation," in International Conference on Advanced Intelligent Mechatronics, 2013, pp. 100-105. 
[6] C. Yamahata, E. Sarajlic, M. Stranczl, G. J. M. Krijnen, and M. A. M. Gijs, "Subpixel Translation of MEMS Measured by Discrete Fourier Transform Analysis of CCD Images," in SolidState Sensors, Actuators and Microsystems Conference, 2011.

[7] P. Masa, E. Franzi, and C. Urban, "Nanometric Resolution Absolute Position Encoder," in Proc. 13th European Space Mechanisms and Tribology Symposium, 2009.

[8] J. Galeano Zea, P. Sandoz, E. Gaiffe, S. Launay, L. Robert, M. Jacquot, F. Hirchaud, J.-L. Pretet, and C. Mougin, "Position-Referenced Microscopy for Live Cell Culture Monitoring," Biomedical Optics Express, vol. 2, no. 5, pp. 1307-1318, 2011.

[9] S. Ri, S. Hayashi, X. Ogihara, and H. Tsuda, "Accurate Full-Field Optical Displacement Measurement Technique using a Digital Camera and Repeated Patterns," Optics Express, vol. 22 , no. 8, pp. 9693-9706, 2014.

[10] H. Sugiura, S. Sakuma, M. Kaneko, and F. Arai, "On-Chip Method to Measure Mechanical Characteristics of a Single Cell by Using Moiré Fringe," Micromachines, vol. 6, no. 6, pp. 660$673,2015$.

[11] K. Creath, "Step Height Measurement using Two-Wavelength Phase-Shifting Interferometry," Applied optics, vol. 26, no. 14, pp. 2810-2816, 1987.

[12] M. Born and E. Wolf, Principles of Optics: Electromagnetic Theory of Propagation, Interference and Diffraction of Light, 7th (expanded) ed. Cambridge university press, 1999.

[13] V. Guelpa, G. J. Laurent, P. Sandoz, J. G. Zea, and C. Clevy, "Subpixelic Measurement of Large 1D Displacements: Principle, Processing Algorithms, Performances and Software," Sensors, vol. 14, no. 3, pp. 5056-5073, 2014.

[14] V. Guelpa, G. Laurent, P. Sandoz, and C. Clevy, "Vision-Based Microforce Measurement With a Large Range-to-Resolution Ratio Using a Twin-Scale Pattern," IEEE Transaction on Mechatronics, vol. 20, no. 6, pp. 3148-3156, 2015.

[15] Joint Committee for Guides on Metrology, International vocabulary of metrology - Basic and general concepts and associated terms (VIM), 3rd edn., 2012.

\section{Biographies}

Valérian Guelpa is graduated from the National School of Mechanics and Microtechnologies (ENSMM) of Besançon in France and received his Master degree of Mechatronics, Microsystems and Embedded Electronics from the University of Franche-Comté in 2013. As a Ph.D. student at the AS2M (Automatic Control and MicroMechatronic Systems) department of FEMTO-ST Institute, his research involves microrobotics and visual measurements.

Patrick Sandoz received his Ph.D. in engineering science from the University of Franche-Comté in France, in 1993. He is currently senior researcher at CNRS, french National Center for Scientific Research, and member of the Applied Mechanics Department of FEMTO-ST Institute in Besançon, France. His research interests include optical instrumentation and measurements and their applications.

Miguel Asmad Vergara studied Physics at the National University of Trujillo in Peru, and received his Magister degree in Applied Physics at the Pontificia Universidad Católica del Perú in 2008, where presently is a Professor of Optics and Laser and its applications. His early research focused on experimental speckle metrology for testing deformation and vibration. At the present time their studies are in high-accurate position monitoring of mobile targets, in a co-supervision doctoral program between the Université de Bourgogne-Franche-Comté, France and the Pontificia Universidad Católica del Perú.

Cédric Clévy was graduated from the Ecole Normale Supérieure de Cachan, France in 2001, received his Master degree of Mechanical engineering, Automatics and $\mathrm{Au}-$ tomation in 2002 and his Ph. D. degree in Automatic Control and computer Sciences in 2005. Since 2006, he has been an Associate Professor at the University of Franche-Comté, Besançon, France working in the AS2M (Automatic Control and MicroMechatronic Systems) department of FEMTO-ST Institute. His research interests are the design, modeling and control of microrobotic and microassembly systems oriented to MOEMS.

Nadine Le-Fort Piat received her Ph.D. in Control Systems and Signal Processing at the University of Technology of Compiègne (UTC) in 1984. She became associate professor in 1984 at UTC and professor in 1998 at the National Engineering School of Mechanics and Microtechnologies (ENSMM) in Besançon She is a member of the Automatic Control and Micro-Mechatronic Systems Department of the FEMTO-ST Institute. Her research areas concern perception and advanced control strategies based on visual servoing for micro and nanomanipulation

Guillaume J. Laurent received his Ph.D. in control systems and computer sciences from the University of FrancheComté in France, in 2002. He is currently associate professor at the National School of Mechanics and Microtechnologies (ENSMM) in Besançon. He is member of the Automatic Control and Micro-Mechatronic Systems Department of FEMTO-ST Institute. His research interests include microrobotics, micromanipulation, distributed manipulation, and visual servoing. 